\title{
Multipoint Model Order Reduction of delayed PEEC systems
}

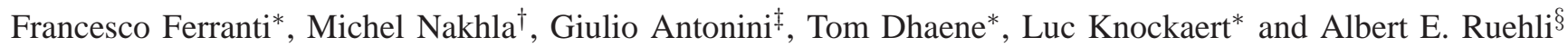 \\ *Department of Information Technology, Ghent University-IBBT, Gaston Crommenlaan 8 Bus 201, B-9050, Ghent, Belgium \\ Email: francesco.ferranti@intec.ugent.be; tom.dhaene@intec.ugent.be; luc.knockaert@intec.ugent.be \\ ${ }^{\dagger}$ Department of Electronics, Carleton University, Ottawa, ON K1S 5B6, Canada \\ E-mail:msn@doe.carleton.ca. \\ ${ }_{\ddagger}^{\ddagger}$ Dipartimento di Ingegneria Elettrica e dell’Informazione, Università degli Studi dell’Aquila, 67100, L'Aquila, Italy \\ Email: giulio.antonini@univaq.it \\ $\S$ Missouri University of S\&T in Rolla \\ E-mail: albert.ruehli@gmail.com
}

\begin{abstract}
We present a new model order reduction technique for electrically large systems with delay elements, which can be modeled by means of neutral delayed differential equations. An adaptive multipoint expansion and model order reduction of equivalent first order systems are combined in the new proposed method that preserves the neutral delayed differential formulation. An adaptive algorithm to select the expansion points is presented. The proposed model order reduction technique is validated by pertinent numerical results. A comparison with a previous model order reduction algorithm based on a single point expansion is performed to show the considerably improved modeling capability of the new proposed technique.

Index Terms-Delayed Partial Element Equivalent Circuit method, model order reduction, neutral delayed differential equations.
\end{abstract}

\section{INTRODUCTION}

Nowadays, the accurate modeling of modern integrated circuits and high-speed systems calls for electromagnetic (EM) 3-D methods [1], [2] as necessary analysis and design tools. These EM methods can produce large systems of equations and model order reduction (MOR) techniques are used to reduce the complexity of EM models and the computational cost of the simulations, while retaining the important physical features of the original system [3], [4]. Among all EM methods, the Partial Element Equivalent Circuit (PEEC) method [2] uses a circuit interpretation of the Electric Field Integral Equation (EFIE) [5], therefore it is able to handle problems with both electromagnetic fields and circuits [2], [6]. Considering a quasi-static PEEC formulation [2], the time delays between the elements in the full-wave PEEC formulation [7] are neglected and an equivalent RLC circuit and systems of ordinary differential equations (ODE) are obtained. Standard MOR techniques for ODE systems can be used to reduce the size of a quasi-static PEEC model [3], [4]. When geometric dimensions become electrically large and the frequency content of signal waveform increases, time delays must be taken into account and, therefore, included in the modeling process.

A PEEC formulation which include delay elements, called $\tau$ PEEC [8], becomes necessary and leads to systems of neutral delayed differential equations (NDDE) [7]. Over the years, several successful MOR methods for large ODE systems have been proposed. Since standard MOR techniques for ODE systems cannot be directly applied to NDDE systems, the reduction of large NDDE systems is still a very challenging research topic. Especially, the reduction of electrically large structures where delays among coupled elements cannot be neglected or easily approximated by rational basis functions needs to be investigated and addressed.

Some techniques for the reduction of NDDE systems have been proposed [9]-[12]. In [9] an equivalent first order system is computed by means of a Taylor expansion, and then MOR Krylov subspace methods [3], [4] are applied. This MOR technique does not preserve the NDDE formulation. The construction of rational local approximations of $\tau$ PEEC systems and multiple expansion points are used in [10]. Then, each rational local approximation is reduced by standard MOR methods and portions of these reduced rational models are combined to obtain a global reduced model for the frequency range of interest. As in [9], the NDDE formulation is not preserved in the reduction process. In [11], some exponential terms (primary phase factors) are extracted and the smoother remainders are expanded into a linear form and then projected to obtain the reduced model. Hence, the extraction of primary phase factors and the segregation of the system into multiple remainder phase matrices are needed. In [12], an equivalent first order system is computed by means of a single point Taylor expansion [9] and a corresponding orthogonal projection matrix is computed by means of a block Arnoldi algorithm [3]. Then, an orthogonal projection matrix for the original NDDE system is extracted and a reduced NDDE system is obtained. 
This paper presents a new MOR method for $\tau$ PEEC models that is able to accurately reduce electrically large structures with delay elements that cannot be neglected or easily approximated by rational basis functions. An adaptive multipoint expansion and MOR of equivalent first order systems [12] are combined in the new proposed technique. The NDDE formulation is preserved in the reduced model.

The paper is organized as follows. Section II describes the modified nodal analysis (MNA) equations of the $\tau$ PEEC method. Section III describes the proposed MOR method for NDDE systems. Section IV presents numerical results based on the $\tau$ PEEC method to validate the proposed MOR technique. A comparison with the model order reduction algorithm based on a single point expansion [12] is performed to show the considerably improved modeling capability of the new proposed technique.

\section{II. $\tau$ PEEC FORMULATION}

The PEEC method [2] stems from the integral equation form of Maxwell's equations and it is able to provide a circuit interpretation of the EFIE equation, thus allowing to handle complex problems involving both circuits and electromagnetic fields.

In the standard approach, volumes and surfaces are discretized into elementary regions, hexahedra and patches respectively [8] over which the current and charge densities are expanded into a series of basis functions.

Nodes and branches are generated and electrical lumped elements are identified modeling both the magnetic and electric field coupling by means of the standard Galerkin's testing procedure.

Conductors are modeled by their ohmic resistance, while dielectrics require modeling the polarization charge due to the dielectric polarization [13]. Partial inductances and coefficients of potential model magnetic and electric field coupling, respectively. The magnetic field coupling between two inductive volume cells $\alpha$ and $\beta$ is modeled by the partial inductance

$$
L p_{\alpha \beta}=\frac{\mu}{4 \pi} \frac{1}{a_{\alpha} a_{\beta}} \int_{u_{\alpha}} \int_{u_{\beta}} \frac{1}{R_{\alpha \beta}} d u_{\alpha} d u_{\beta}
$$

where $R_{\alpha \beta}$ is the distance between any two points in volumes $u_{\alpha}$ and $u_{\beta}$ with $a_{\alpha}$ and $a_{\beta}$ their cross sections. The electric field coupling between two capacitive surface cells $\gamma$ and $\delta$ is modeled by the coefficient of potential

$$
P_{\gamma \delta}=\frac{1}{4 \pi \varepsilon} \frac{1}{S_{\gamma} S_{\delta}} \int_{S_{\gamma}} \int_{S_{\delta}} \frac{1}{R_{\gamma \delta}} d S_{\gamma} d S_{\delta}
$$

where $R_{\gamma \delta}$ is the distance between any two points on surfaces $\gamma$ and $\delta$, while $S_{\gamma}$ and $S_{\delta}$ denote the area of their respective surfaces.

Due to the finite value of the speed of light, partial inductances and coefficients of potentials relate causes and effects delayed in time:

$$
\begin{aligned}
& v_{L, \alpha}(t)=L p_{\alpha \beta} \frac{d i_{\beta}\left(t-\tau_{\alpha \beta}\right)}{d t} \\
& v_{P, \gamma}(t)=P_{\gamma \delta} q_{\delta}\left(t-\tau_{\gamma \delta}\right)
\end{aligned}
$$

where $\tau_{\alpha \beta}=R_{\alpha \beta} / c_{0}$ and $\tau_{\gamma \delta}=R_{\gamma \delta} / c_{0}$ are the centerto-center propagation times between the corresponding basisfunction domains, and $c_{0}$ denotes the free-space speed of light. Hence, partial inductance and coefficient of potential matrices act as a delay operator for time derivatives of currents and charges, respectively

$$
\begin{aligned}
\mathbf{v}_{L}(t) & =\mathbf{L}_{p} \frac{d \mathbf{i}\left(t-\boldsymbol{\tau}_{L}\right)}{d t}=\widetilde{\mathbf{L}}_{p}\left(\boldsymbol{\tau}_{L}\right) \frac{d \mathbf{i}(t)}{d t} \\
\mathbf{v}(t) & =\mathbf{P q}\left(t-\boldsymbol{\tau}_{C}\right)=\widetilde{\mathbf{P}}\left(\boldsymbol{\tau}_{C}\right) \mathbf{q}(t)
\end{aligned}
$$

where $\tau_{L}$ and $\tau_{C}$ denote the center-to-center delay matrices for the magnetic and electric field couplings, respectively.

Generalized Kirchoff's laws, for conductors, can be rewritten as

$$
\begin{aligned}
& \frac{d \mathbf{q}(t)}{d t}-\mathbf{A}^{T} \mathbf{i}(t)+\mathbf{i}_{e}(t)=0 \\
& -\mathbf{A v}(t)-\widetilde{\mathbf{L}}_{p}\left(\boldsymbol{\tau}_{L}\right) \frac{d \mathbf{i}(t)}{d t}-\mathbf{R i}(t)=\mathbf{0}
\end{aligned}
$$

where $\mathbf{A}$ is the connectivity matrix, $\mathbf{v}(t)$ denotes the node potentials to infinity, $\mathbf{i}(t)$ and $\mathbf{i}_{e}(t)$ represent the currents flowing in volume cells and the external currents, respectively.

The previous equation (6b) has to be modified when dielectrics are considered, since the resistance $\mathbf{R}$ is substituted by the excess capacitance which relates the polarization charge and the corresponding voltage drop as $\mathbf{v}_{d}(t)=\mathbf{C}_{d}^{-1} \mathbf{q}_{d}(t)$ [13]. Hence, for dielectric elementary cells, (6) become

$$
\begin{aligned}
& \frac{d \mathbf{q}(t)}{d t}-\mathbf{A}^{T} \mathbf{i}(t)+\mathbf{i}_{e}(t)=0 \\
& -\mathbf{A v}(t)-\widetilde{\mathbf{L}}_{p}\left(\boldsymbol{\tau}_{L}\right) \frac{d \mathbf{i}(t)}{d t}-\mathbf{v}_{d}(t)=\mathbf{0} \\
& \mathbf{i}(t)=\mathbf{C}_{d} \frac{d \mathbf{v}_{d}(t)}{d t}
\end{aligned}
$$

A selection matrix $\mathbf{K}$ is introduced to define the port voltages by selecting node potentials. The same matrix is used to obtain the external currents $\mathbf{i}_{e}(t)$ by the currents $\mathbf{i}_{s}(t)$ which are of opposite sign with respect to the port currents $\mathbf{i}_{p}(t)$

$$
\begin{aligned}
\mathbf{v}_{p}(t) & =\mathbf{K} \mathbf{v}(t) \\
\mathbf{i}_{e}(t) & =\mathbf{K}^{T} \mathbf{i}_{s}(t) .
\end{aligned}
$$

An example of $\tau$ PEEC circuit for a conductor elementary cell is illustrated, in the Laplace domain, in Fig. 1 where the current controlled voltage sources $s L_{p, i j} I_{j}$ and the charge controlled current sources $d Q_{i} / d t$ model the magnetic and electric field couplings, respectively. 


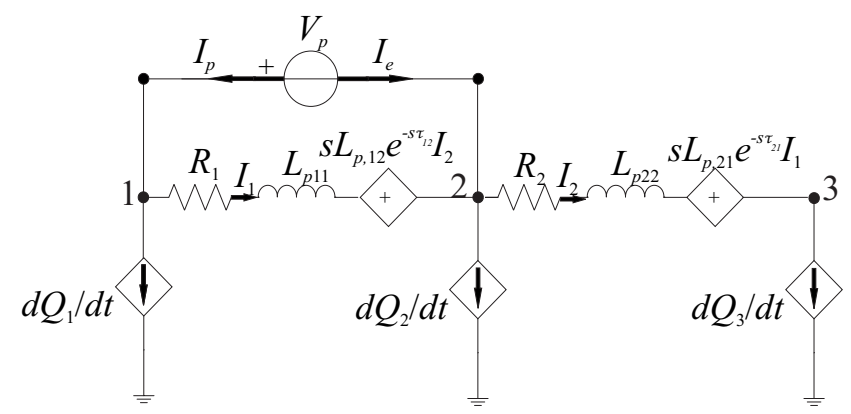

Fig. 1. Illustration of $\tau$ PEEC circuit electrical quantities for a conductor elementary cell.

\section{Descriptor representation of PEEC circuits}

We assume that the system under analysis consists of conductors and dielectrics. Let the current and charge densities be defined in volumes and surface of conductors and dielectrics, respectively. The Galerkin approach is applied to convert the continuous electromagnetic problem described by the EFIE to a discrete problem in terms of electrical circuit quantities. Let us denote with $n_{n}$ the number of nodes and $n_{i}$ the number of branches where currents flow. Among the latter, we denote with $n_{c}$ and $n_{d}$ the number of branches of conductors and dielectrics, respectively. Furthermore, let us assume to be interested in generating an admittance representation having $n_{p}$ output currents $\mathbf{i}_{p}(t)$ under voltage excitation $\mathbf{v}_{p}(t)$. Since dielectrics require the excess capacitance to model the polarization charge [13], additional $n_{d}$ unknowns are needed in addition to currents. Hence, if the MNA approach [14] is used, the global number of unknowns is $n_{u}=n_{i}+n_{d}+n_{n}+n_{p}$. In a matrix form, (6)-(8), taking (5) into account, read

$$
\begin{aligned}
& \underbrace{\left[\begin{array}{cccc}
\mathbf{I}_{n_{n}, n_{n}} & \mathbf{0}_{n_{n}, n_{i}} & \mathbf{0}_{n_{n}, n_{d}} & \mathbf{0}_{n_{n}, n_{p}} \\
\mathbf{0}_{n_{i}, n_{n}} & \widetilde{\mathbf{L}}_{p}\left(\boldsymbol{\tau}_{L}\right) & \mathbf{0}_{n_{i}, n_{d}} & \mathbf{0}_{n_{i}, n_{p}} \\
\mathbf{0}_{n_{d}, n_{n}} & \mathbf{0}_{n_{d}, n_{i}} & \mathbf{C}_{d} & \mathbf{0}_{n_{d}, n_{p}} \\
\mathbf{0}_{n_{p}, n_{n}} & \mathbf{0}_{n_{p}, n_{i}} & \mathbf{0}_{n_{p}, n_{d}} & \mathbf{0}_{n_{p}, n_{p}}
\end{array}\right]}_{\mathbf{C}} d \underbrace{\left[\begin{array}{c}
\mathbf{q}(t) \\
\mathbf{i}(t) \\
\mathbf{v}_{d}(t) \\
\mathbf{i}_{s}(t)
\end{array}\right]}_{\mathbf{x}(t)}= \\
& \underbrace{\left[\begin{array}{cccc}
\mathbf{0}_{n_{n}, n_{n}} & -\mathbf{A}^{T} & \mathbf{0}_{n_{n}, n_{d}} & \mathbf{K}^{T} \\
\mathbf{A} \widetilde{\mathbf{P}}\left(\boldsymbol{\tau}_{C}\right) & \mathbf{R} & \boldsymbol{\Phi} & \mathbf{0}_{n_{i}, n_{p}} \\
\mathbf{0}_{n_{d}, n_{n}} & -\boldsymbol{\Phi}^{T} & \mathbf{0}_{n_{d}, n_{d}} & \mathbf{0}_{n_{d}, n_{p}} \\
-\mathbf{K} \widetilde{\mathbf{P}}\left(\boldsymbol{\tau}_{C}\right) & \mathbf{0}_{n_{p}, n_{i}} & \mathbf{0}_{n_{p}, n_{d}} & \mathbf{0}_{n_{p}, n_{p}}
\end{array}\right]}_{\mathbf{G}} \cdot \underbrace{\left[\begin{array}{c}
\mathbf{q}(t) \\
\mathbf{i}(t) \\
\mathbf{v}_{d}(t) \\
\mathbf{i}_{s}(t)
\end{array}\right]}_{\mathbf{x}(t)}+ \\
& \underbrace{\left[\begin{array}{c}
\mathbf{0}_{n_{n}+n_{i}+n_{d}, n_{p}} \\
-\mathbf{I}_{n_{p}, n_{p}}
\end{array}\right]}_{\mathbf{B}} \cdot \underbrace{\left[\mathbf{v}_{p}(t)\right]}_{\mathbf{u}(t)}
\end{aligned}
$$

where $\mathbf{I}_{n_{p}, n_{p}}$ is the identity matrix of dimensions equal to the number of ports. Matrix $\boldsymbol{\Phi}$ is

$$
\boldsymbol{\Phi}=\left[\begin{array}{l}
\mathbf{0}_{n_{c}, n_{d}} \\
\mathbf{I}_{n_{d}, n_{d}}
\end{array}\right]
$$

If the delay operator $\widetilde{\mathbf{P}}\left(\boldsymbol{\tau}_{C}\right)$ is applied to the first equation in (9), the system can be recast as

$$
\begin{aligned}
& \underbrace{\left[\begin{array}{cccc}
\widetilde{\mathbf{P}}\left(\boldsymbol{\tau}_{C}\right) & \mathbf{0}_{n_{n}, n_{i}} & \mathbf{0}_{n_{n}, n_{d}} & \mathbf{0}_{n_{n}, n_{p}} \\
\mathbf{0}_{n_{i}, n_{n}} & \widetilde{\mathbf{L}}_{p}\left(\boldsymbol{\tau}_{L}\right) & \mathbf{0}_{n_{i}, n_{d}} & \mathbf{0}_{n_{i}, n_{p}} \\
\mathbf{0}_{n_{d}, n_{n}} & \mathbf{0}_{n_{d}, n_{i}} & \mathbf{C}_{d} & \mathbf{0}_{n_{d}, n_{p}} \\
\mathbf{0}_{n_{p}, n_{n}} & \mathbf{0}_{n_{p}, n_{i}} & \mathbf{0}_{n_{p}, n_{d}} & \mathbf{0}_{n_{p}, n_{p}}
\end{array}\right]}_{\mathbf{C}} \frac{d}{d t} \underbrace{\left[\begin{array}{c}
\mathbf{q}(t) \\
\mathbf{i}(t) \\
\mathbf{v}_{d}(t) \\
\mathbf{i}_{s}(t)
\end{array}\right]}_{\mathbf{x}(t)}= \\
& -\underbrace{\left[\begin{array}{cccc}
\mathbf{0}_{n_{n}, n_{n}} & -\widetilde{\mathbf{P}}\left(\boldsymbol{\tau}_{C}\right) \mathbf{A}^{T} & \mathbf{0}_{n_{n}, n_{d}} & \widetilde{\mathbf{P}}\left(\boldsymbol{\tau}_{C}\right) \mathbf{K}^{T} \\
\mathbf{A} \widetilde{\mathbf{P}}\left(\boldsymbol{\tau}_{C}\right) & \mathbf{R} & \boldsymbol{\Phi} & \mathbf{0}_{n_{i}, n_{p}} \\
\mathbf{0}_{n_{d}, n_{n}} & -\boldsymbol{\Phi}^{T} & \mathbf{0}_{n_{d}, n_{d}} & \mathbf{0}_{n_{d}, n_{p}} \\
-\mathbf{K} \widetilde{\mathbf{P}}\left(\boldsymbol{\tau}_{C}\right) & \mathbf{0}_{n_{p}, n_{i}} & \mathbf{0}_{n_{p}, n_{d}} & \mathbf{0}_{n_{p}, n_{p}}
\end{array}\right]}_{\mathbf{G}} . \\
& \underbrace{\left[\begin{array}{c}
\mathbf{q}(t) \\
\mathbf{i}(t) \\
\mathbf{v}_{d}(t) \\
\mathbf{i}_{s}(t)
\end{array}\right]}_{\mathbf{x}(t)}+\underbrace{\left[\begin{array}{c}
\mathbf{0}_{n_{n}+n_{i}+n_{d}, n_{p}} \\
-\mathbf{I}_{n_{p}, n_{p}}
\end{array}\right]}_{\mathbf{B}} \cdot \underbrace{\left[\mathbf{v}_{p}(t)\right]}_{\mathbf{u}(t)}
\end{aligned}
$$

In a more compact form, (11) can be rewritten as

$$
\begin{aligned}
\mathbf{C}(\boldsymbol{\tau}) \frac{d \mathbf{x}(t)}{d t} & =-\mathbf{G}(\boldsymbol{\tau}) \mathbf{x}(t)+\mathbf{B u}(t) \\
\mathbf{i}_{p}(t) & =\mathbf{L}^{T} \mathbf{x}(t)
\end{aligned}
$$

where $\mathbf{x}(t) \in \Re^{n_{u} \times 1}$ and $\boldsymbol{\tau} \in \Re^{n_{\tau} \times 1}$ contains all delays $\boldsymbol{\tau}_{L}, \boldsymbol{\tau}_{C}$, which denote the center-to-center delay matrices for the magnetic and electric field coupling. Since this is an $n_{p^{-}}$ port formulation, whereby the only sources are the voltage sources at the $n_{p}$-port nodes, $\mathbf{B}=\mathbf{L}$ where $\mathbf{B} \in \Re^{n_{u} \times n_{p}}$. Each delayed entry of matrices $\mathbf{C}(\boldsymbol{\tau})$ and $\mathbf{G}(\boldsymbol{\tau})$ act as a delay operator for the corresponding entry of vector $\mathbf{x}(t)$. Hence, (12) can be re-written in the Laplace domain as:

$$
\begin{aligned}
s \mathbf{C}(s) \mathbf{X}(s) & =-\mathbf{G}(s) \mathbf{X}(s)+\mathbf{B V}_{p}(s) \\
\mathbf{I}_{p}(s) & =\mathbf{B}^{T} \mathbf{X}(s) \\
\mathbf{C}(s) & =\mathbf{C}_{0}+\sum_{k=1}^{n_{\tau}} \mathbf{C}_{k} e^{-s \tau_{k}} \\
\mathbf{G}(s) & =\mathbf{G}_{0}+\sum_{k=1}^{n_{\tau}} \mathbf{G}_{k} e^{-s \tau_{k}}
\end{aligned}
$$

\section{Single Point AND MULTiPoint DELAYED Model ORDER REDUCTION}

In [12], an equivalent first order system is computed by means of a single point Taylor expansion [9] and a corresponding orthogonal projection matrix is computed by means of a block Arnoldi algorithm [3]. Then, an orthogonal projection matrix for the original NDDE system is extracted and a reduced NDDE system is obtained. The NDDE formulation is preserved in the reduction process. The equivalent first order system obtained after the single point Taylor expansion of exponential terms has an order equal to $q n_{u}$, where $q$ is the order of the Taylor expansion and $n_{u}$ the order of the original NDDE system [12]. The reduction of equivalent first order 
Algorithm 1: $\operatorname{Multipoint}\left(N D D E_{\text {orig }},\left\{s_{k}\right\}_{k=1}^{n_{\text {points }}}, n_{r}, q\right)$

comment: Multipoint computation of basis $\mathbf{Q}$

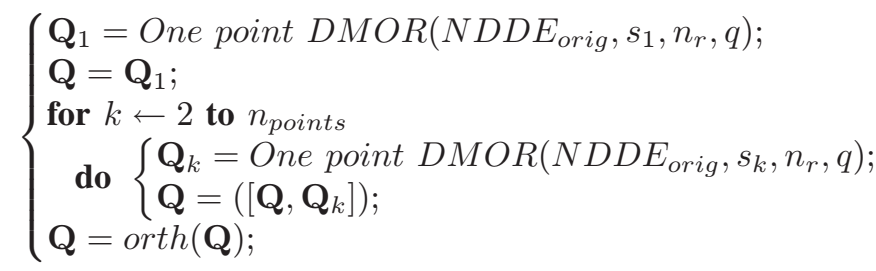

Fig. 2. Pseudocode for computing the orthogonal basis $\mathbf{Q}$ in the multipoint expansion case.

systems becomes computationally expensive and sometimes not feasible, when large delays $\left(2 \pi f r e q_{\max } \tau_{\max }>10\right)$ [11] are involved, since exponential terms with large delays need many terms in the Taylor expansion to be accurately approximated. The multipoint feature [15] addresses this issue and is able to accurately reduce NDDE systems with large delays, since a small expansion Taylor order can be used for each expansion point and the accuracy of the reduced model is increased by adding new expansion points. An adaptive multipoint expansion and MOR of equivalent first order systems are used in the proposed novel MOR algorithm. As in [12], the NDDE formulation is preserved. Assuming that the order of the Taylor expansion is fixed for each expansion point, an adaptive algorithm is used to choose the expansion points. At each expansion point, the MOR algorithm described in [12] is applied and the corresponding projection matrix $\mathbf{Q}_{i}, i=1, \ldots, n_{\text {points }}$ is computed. The final projection matrix $\mathbf{Q}$ is based on the orthogonalization of the stack column collection of all single expansion point projection matrices. The computation of $\mathbf{Q}$ in the case of multipoint expansion is explained in the flowchart in Fig. 2. The MOR algorithm described in [12] is called One point DMOR in Fig. 2, where $n_{r}=q n_{p}$ represents the reduced order for each expansion point.

Once $\mathbf{Q}$ is computed, it is applied to the original NDDE system (13)-(14) and a reduced NDDE system

$$
\begin{aligned}
s \mathbf{C}_{r}(s) \boldsymbol{\chi}(s) & =-\mathbf{G}_{r}(s) \boldsymbol{\chi}(s)+\mathbf{B}_{r} \mathbf{V}_{p}(s) \\
\mathbf{I}_{p}(s) & =\mathbf{B}_{r}^{T} \boldsymbol{\chi}(s) \\
\mathbf{C}_{r}(s) & =\mathbf{C}_{r, 0}+\sum_{k=1}^{n_{\tau}} \mathbf{C}_{r, k} e^{-s \tau_{k}} \\
\mathbf{G}_{r}(s) & =\mathbf{G}_{r, 0}+\sum_{k=1}^{n_{\tau}} \mathbf{G}_{r, k} e^{-s \tau_{k}}
\end{aligned}
$$

is obtained, where the following congruence transformations are used

$$
\begin{aligned}
\mathbf{C}_{r, i} & =\mathbf{Q}^{T} \mathbf{C}_{i} \mathbf{Q}, i=0, \ldots, n_{\tau} \\
\mathbf{G}_{r, i} & =\mathbf{Q}^{T} \mathbf{G}_{i} \mathbf{Q}, i=0, \ldots, n_{\tau} \\
\mathbf{B}_{r} & =\mathbf{Q}^{T} \mathbf{B} \\
\mathbf{L}_{r} & =\mathbf{Q}^{T} \mathbf{L}
\end{aligned}
$$

\section{Algorithm for the selection of expansion points}

Assuming that the order of the Taylor expansion $q$ is fixed for each expansion point, an algorithm is needed to choose the location of the expansion points. An adaptive and iterative algorithm is proposed to determine the expansion points. It is based on an iterative comparison between reduced and original model. It starts from two expansion points $s_{\text {min }}=j \omega_{\text {min }}$, $s_{\max }=j \omega_{\max }$ located at the minimum and maximum frequency of interest and the corresponding reduced model is compared with the original model in the midpoint between $s_{\min }$ and $s_{\max }$, therefore $s_{\text {mid,1 }}=\frac{s_{\min }+s_{\max }}{2}$. If an error threshold is satisfied, then the algorithm stops, otherwise this midpoint is considered as a new expansion point and a new reduced model is computed, which contain information from all the expansion points $s_{\min }, s_{\operatorname{mid}, 1}, s_{\max }$, and it is compared with the original model in two new points, namely the midpoints of the intervals $\left[s_{\text {min }}, s_{\text {mid,1 }}\right]$ and $\left[s_{\text {mid,1 }, 1}, s_{\text {max }}\right]$. If no comparison point exceeds the error threshold, then the algorithm stops, otherwise the new expansion points for the next iteration are chosen as the comparison points that exceed the error threshold. If some comparison points satisfy the error threshold, the corresponding intervals defined by expansion points are considered to be accurately described and are not checked for accuracy in the next iterations. The comparison points at each iteration are the midpoints of all intervals defined by expansion points, except those ones considered accurate in the previous iterations. Concerning the error criterion, let us define the weighted RMS-error as

$$
\begin{aligned}
& \operatorname{Err}= \\
& =\sqrt{\frac{\sum_{i=1}^{\left(n_{p}\right)^{2}} \sum_{k=1}^{K_{s}} \mid w_{Y_{i}}\left(s_{k}\right)\left(Y_{r, i}\left(s_{k}\right)-\left.Y_{i}\left(s_{k}\right)\right|^{2}\right.}{\left(n_{p}\right)^{2} K_{s}}}
\end{aligned}
$$

with

$$
w_{Y_{i}}(s)=\left|\left(Y_{i}(s)\right)^{-1}\right|
$$

In the comparison step at each iteration, the error is computed on each comparison point separately, therefore $K_{s}=1$ and the accuracy threshold is chosen equal to 0.05 .

\section{NUMERICAL RESULTS}

A numerical example compares the approach described in [12] with the new proposed approach. It shows that the new MOR technique is able to accurately reduce a system with large delays, while the technique in [12], based on a single point expansion, does not perform well. The proposed MOR algorithm was implemented in Matlab R2009A [16] and all 
experiments were carried out on Windows platform on Intel Core2 Extreme CPU Q9300 2.53GHz machines with 8GB RAM.

\section{Three Wires with Bend}

A system composed of three long, parallel, bent wires terminating on the same vertical plane is modeled in this example. It is shown in Fig. 3. The system is analyzed on the frequency range $[0.0001,5] \mathrm{GHz}$. The three ports of the system are defined between conductors $1-4,2-5$ and $3-6$.

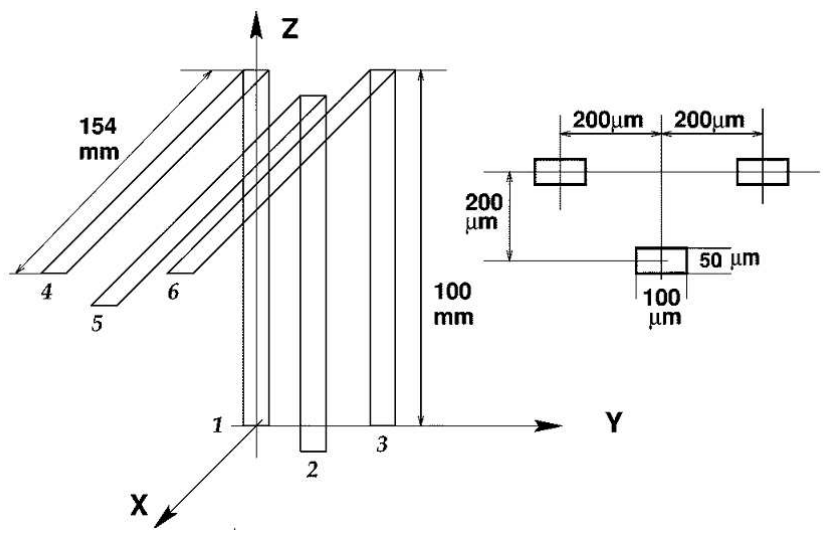

Fig. 3. Three wires with bend.

The order of the original $\tau$ PEEC model is equal to $n_{u}=$ 3039 , the number of delays is equal to 899 and the largest delay gives $2 \pi f r e q_{\max } \tau_{\max }=19.2$. Table I shows some parameters of the new proposed MOR method and the MOR method described in [12].

TABLE I

MOR PARAMETERS

\begin{tabular}{|l|c|c|}
\hline Parameter & Multipoint expansion & Single point expansion \\
\hline Taylor terms & 3 & 3 \\
Expansion points & 25 & 1 \\
Reduced order & 450 & 18 \\
Error reduced model & 0.03 & 2.60 \\
\hline
\end{tabular}

Figs. 4-7 compare the magnitude and phase of the original and reduced NDDE models of $\mathbf{Y}_{11}(s)$ and $\mathbf{Y}_{13}(s)$ obtained by the new proposed MOR method and the MOR method presented in [12]. The number of moments matched per expansion point has been chosen equal to 3 , since a higher order leads to an equivalent first order system that cannot be handled by the computer used for the simulations due to RAM limitation. As clearly seen, the presented MOR method can reduce a large NDDE system with large delay terms, while accurately preserving the behavior of the original system. The single point expansion method presented in [12] is not able to accurately reduce the original NDDE system and the new proposed method overcomes its limitations.

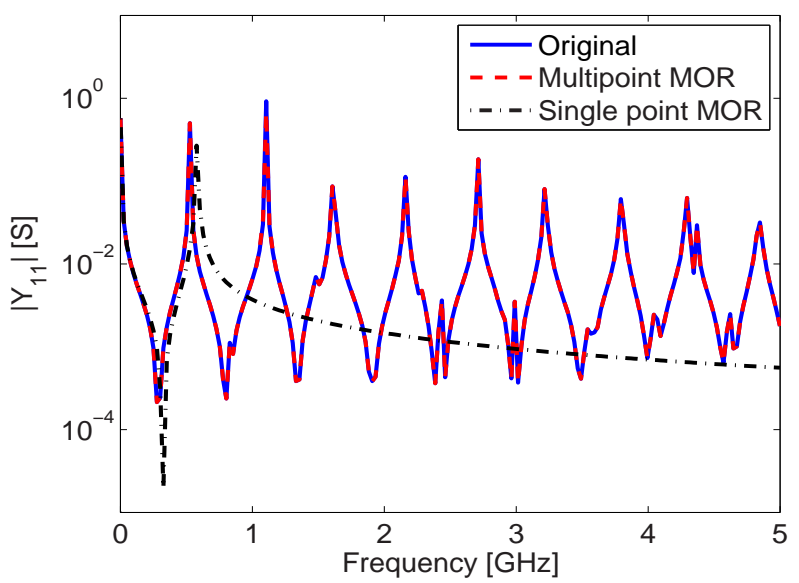

Fig. 4. Magnitude of $\mathbf{Y}_{11}$.

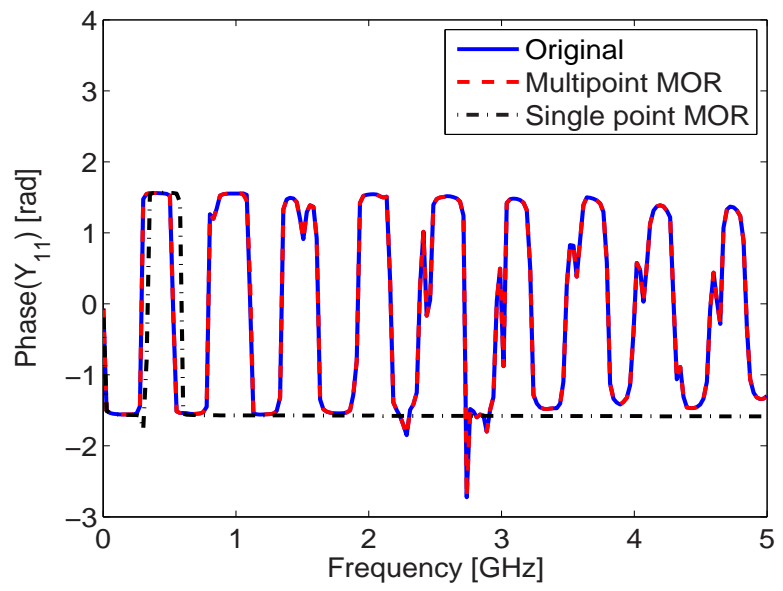

Fig. 5. Phase of $\mathbf{Y}_{11}$.

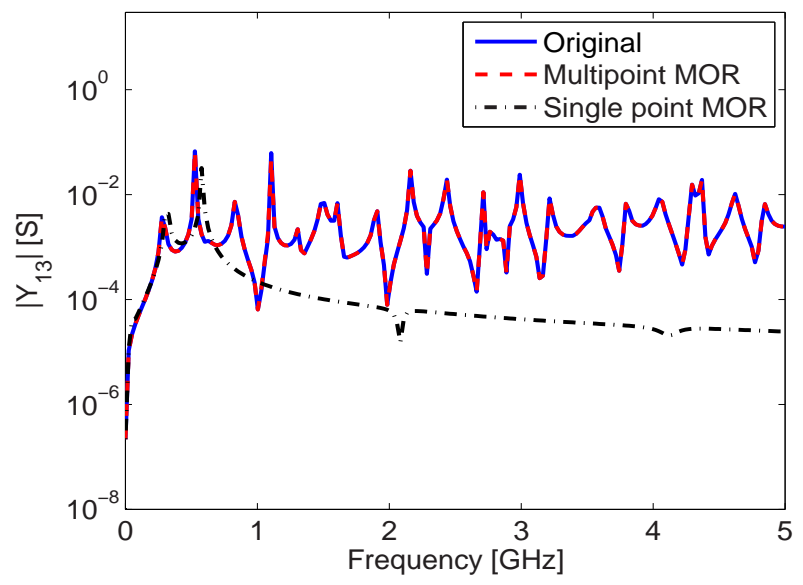

Fig. 6. Magnitude of $\mathbf{Y}_{13}$.

\section{CONCLUSIONS}

We have proposed a new model order reduction technique for large NDDE systems, which is applicable to $\tau$ PEEC 


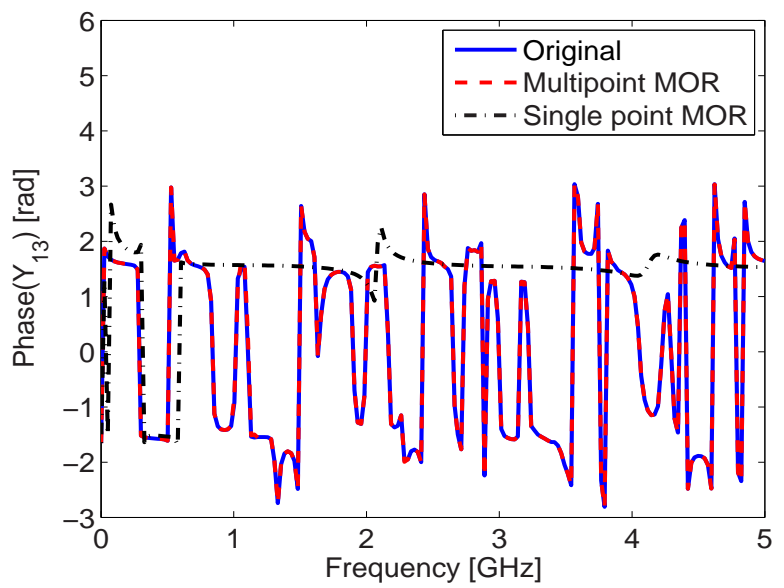

Fig. 7. Phase of $\mathbf{Y}_{13}$.

models. It is able to accurately reduce electrically large structures where delays among coupled elements cannot be neglected or easily approximated by rational basis functions. An adaptive multipoint expansion and MOR of equivalent first order systems are combined in the new proposed MOR method. The NDDE formulation is preserved in the reduced model. Numerical results based on the $\tau$ PEEC method have validated the proposed MOR approach.

\section{ACKNOWLEDGMENT}

This work was supported by the Research Foundation Flanders (FWO).

\section{REFERENCES}

[1] R. F. Harrington, Field Computation by Moment Methods. New York: Macmillan, 1968.

[2] A. E. Ruehli, "Equivalent circuit models for three dimensional multiconductor systems," IEEE Trans. Microw. Theory Tech., vol. 22, no. 3, pp. 216-221, Mar. 1974.

[3] A. Odabasioglu, M. Celik, and L. T. Pileggi, "PRIMA: passive reducedorder interconnect macromodeling algorithm," IEEE Trans. Comput.Aided Design Integr. Circuits Syst., vol. 17, no. 8, pp. 645-654, Aug. 1998.

[4] L. Knockaert and D. De Zutter, "Laguerre-SVD reduced-order modeling," IEEE Trans. Microw. Theory Tech., vol. 48, no. 9, pp. 1469-1475, Sep. 2000

[5] C. A. Balanis, Advanced Engineering Electromagnetics. John Wiley and Sons, New York, 1989.

[6] A. E. Ruehli and A. C. Cangellaris, "Progress in the methodologies for the electrical modeling of interconnects and electronic packages," Proc. IEEE, vol. 89, no. 5, pp. 740-771, May 2001.

[7] P. J. Restle, A. Ruehli, S. G. Walker, G. Papadopoulos, "Full-wave PEEC time-domain for the modeling of on-chip interconnects," IEEE Transactions on Computer-Aided Design, vol. 20, no. 7, pp. 877-887, July 2001.

[8] A. E. Ruehli, G. Antonini, J. Esch, J. Ekman, A. Mayo and A. Orlandi, "Non-orthogonal PEEC formulation for time and frequency domain EM and circuit modeling," IEEE Trans. Electromagn. Compat., vol. 45, no. 2, pp. 167-176, May 2003.

[9] J. R. Phillips, E. Chiprout, and D. D. Ling, "Efficient full-wave electromagnetic analysis via model-order reduction of fast integral transforms," in DAC '96: Proceedings of the 33rd annual Design Automation Conference, New York, NY, USA, 1996, pp. 377-382.
[10] J. Cullum, A. Ruehli, T. Zhang, "A method for reduced-order modeling and simulation of large interconnect circuits and its application to PEEC models with retardation," IEEE Trans. Circuits Syst. II, vol. 47, no. 4, pp. 261-373, Apr. 2000.

[11] T. Klemas, L. Daniel, and J. White, "Segregation by primary phase factors: a full-wave algorithm for model order reduction," in Design Automation Conference, 2005. Proceedings. 42nd, Jun. 2005, pp. $943-$ 946.

[12] W. Tseng, C. Chen, E. Gad, M. Nakhla, and R. Achar, "Passive order reduction for RLC circuits with delay elements," IEEE Transactions on Advanced Packaging, vol. 30, no. 4, pp. 830 -840, Nov. 2007.

[13] A. E. Ruehli and H. Heeb, "Circuit models for three-dimensional geometries including dielectrics," IEEE Trans. Microw. Theory Tech., vol. 40, no. 7, pp. 1507-1516, Jul. 1992.

[14] C. Ho, A. Ruehli, P. Brennan, "The modified nodal approach to network analysis," IEEE Trans. Circuits Syst., vol. 22, no. 6, pp. 504-509, Jun. 1975 .

[15] I. Elfadel and D. Ling, "A block rational Arnoldi algorithm for multipoint passive model-order reduction of multiport RLC networks," in IEEE/ACM International Conference on Computer-Aided Design, Nov. 1997, pp. $66-71$.

[16] “Matlab User's Guide," The Mathworks, Inc., Natick, 2009. 